\title{
Comprehensive Analysis of Universal Stress Proteins and their Promoter Sequences in Rice
}

\author{
Rakesh Bhowmick* \\ ICAR-Vivekananda Parvatiyakrishi Anusandhan Sansthan, Almora-263601, \\ Uttarakhand, India \\ ICAR-Indian Agricultural Research Institute, New Delhi-110012, India \\ *Corresponding author
}

\begin{tabular}{|l|}
\hline Ke y w o r d s \\
Universal stress \\
proteins, \\
$\begin{array}{l}\text { Promoter sequences } \\
\text { in rice }\end{array}$ \\
\hline Article Info \\
\hline $\begin{array}{l}\text { Accepted: } \\
\text { 12 June } 2019 \\
\text { Available Online: } \\
\text { 10 July } 2019\end{array}$ \\
\hline
\end{tabular}

\section{A B S T R A C T}

Rice is one of the important cereal crops and more than half of the world population is dependent on Rice as staple food. Rice cultivation is affected by various abiotic stresses like drought, salinity, cold etc. Universal Stress Proteins $(U S P \mathrm{~s})$ are conserved proteins found across the plant, animals, fungi and bacteria. USPs are reported to be involved in various abiotic stress responses. In this study we have identified 43 USP genes in rice, distributed across the chromosome. Phylogenetic relationship between OSUSP protein sequences revealed four distinct groups, whereas phylogenetic tree of OSUSP promoter showed presence of six groups. Gene expression of $O s U S P \mathrm{~s}$ in response to various abiotic stresses showed differential expression pattern in different stresses. We have also analysed promoter sequence of $O S U S P$ s to find potential transcription factor binding site. We found 14533 TFBS in 43 OsUSP promoter region, which belong to 41 transcription factor family.

\section{Introduction}

Universal Stress Proteins (USPs) are highly conserved, found across most of the living organisms including bacteria, archea, fungi, plants and mammals (Kerk et al., 2003; Ndimba et al., 2005, Persson et al., 2007). Initially the gene was first identified in a bacteria and it was named C13.5proteindue to its mobility in two dimensional gel electrophoresis. Later the name of these proteins was changed to Universal Stress Protein to reflect their responsiveness to cellular stress (Zarembinski et al., 1998; Sousa and McKay, 2001). USPs are identified on the basis of presence of highly conserved 140-160 residues (PF00582). E. coli genome codes for six USPs and they are known to be involved in diverse biological processes including motility, adhesion, and resistance to oxidative stress (Nachin et al., 2005). Orthologs of bacterial $U S P$ s are ubiquitously present in 
plant genome. Arabidopsis and Barley genome is known to encode 44 and 66 USPs respectively (Kerk et al., 2003; Li et al., 2010).USP genes belong to two classes: ATP binding and ATP non-binding (Tkaczuk et al., 2013). Though this gene family has been characterised in several plant species but function of USPs are still not completely understood. USPs play important role in various abiotic stresses. SpUSP was reported to reduce stomatal aperture to minimise harmful effect of drought stress (Rachid et al., 2012). Arabidopsis USP gene, At3g53990 has chaperone function and expression is induced by heat and drought stress (Isokpehi et al., 2012). Another ATP binding AtUSP gene, At3g62550 is upregulated during drought stress. Arabidopsis USPs, AtPHOS32 and AtPHOS34 were found to be phosphorylated during microbial infection (Shinozaki and Yamaguchi-Shinozaki, 2007). Sauter et al., (2002) demonstrated OsUSP1 is involved in activation of signalling cascade in response to ethylene during hypoxia. The USP genes of Gossypium arboretum, Astragalus sinicus, Solanum pennellii, and Salicornia brachiate were shown to be involved in water stress, nodulation, and drought, salt, and osmotic tolerances (Chou et al., 2007; Maqbool et al., 2009; Loukehaich et al., 2012; Udawat et al., 2014). Jung et al., (2015) overexpressed AtUSP to show its role in heat and oxidative stress tolerance. In addition to stress response of USP genes, At $U S P$ promoter was shown to upregulate GUS expression during ABA, ACC, dehydration, heat, cold, salt, and osmotic stress (Bhuria et al., 2016).

Rice is one of the major cereal crops cultivated throughout the world. Rice cultivation is affected by various biotic and abiotic stresses. No comprehensive study has been made till date to analyse promoter region of rice USP genes. In this study, we have identified 44 different $O s U S P$ genes and we have identified common transcription factor binding site within promoters of OsUSPs. Gene expression of $O s U S P$ s was analysed and distribution of $O s U S P$ s across chromosomes was shown using mapchart software.

\section{Materials and Methods}

\section{Identification of $O s U S P s$}

For identification of candidate USP genes in rice, Basic Local Alignment Search Tool (BLAST) and Hidden Markov Model (HMM) search both was performed. Predicted protein sequences of rice were retrieved from ensemble plant database (https://plants.ensembl.org/index.html).

Thirty-eight predicted Arabidopsis USP protein sequences were retrieved from TAIR database. Hidden Markov Model (HMM) profile of USP domain (PF00582) was fetched from PFam database (http://pfam.sanger.ac.uk/) and HMM search was employed against the local database of rice proteins using Hmmer 3.0 ( $e<1 \mathrm{e}-5)$. As HMM profile search might fail to identify protein sequences containing incomplete USP domain, standalone BLASTP search was performed using Arabidopsis USP proteins as query against the respective rice proteins. Evalue less than 1e-5 and percentage identity more than 50 was used as a threshold for further characterization. To confirm the results obtained using the HMMER algorithm and BLASTP search, the putative USP protein sequences were also queried against the Pfam and CDD (http://www.ncbi.nlm.nih.gov/cdd/) databases.

\section{Phylogenetic analysis}

The evolutionary history of OsUSP was inferred using the Neighbor-Joining method. The bootstrap consensus tree inferred from 1000 replicates is taken to represent the evolutionary history of the OsUSP. The evolutionary distances were computed using 
the Poisson correction method and are in the units of the number of amino acid substitutions per site. The analysis involved 43 amino acid sequences. All ambiguous positions were removed for each sequence pair. There were a total of 105 positions in the final dataset. Evolutionary analyses were conducted in MEGA7 and Neighbor-Joining tree was reconstructed. Promoter sequences of OsUSPs were also retrieved and phylogenetic analysis of OsUSP promoters was also performed using neighbour-Joining method. Physical position of OsUSPs was fetched by BioMart data mining tool from Phytozome database. Chromosomal distribution of OsUSPs was visualised by MapChart software.

\section{Promoter analysis of $O s U S P s$}

For analysis of $O s U S P$ gene promoters, $1 \mathrm{~Kb}$ upstream sequence of all the OsUSPs were retrieved by using BioMart. For identification of transcription factor binding site within promoter, PlantPAN 2.0 software (http:// plantpan2.itps.ncku.edu.tw/) was used.

As USP genes are differentially expressed during abiotic stresses, we identified common transcription factor binding sites in promoters, using gene group analysis in PlantPAN 2.0 web tool.

\section{Digital gene expression analysis}

For gene expression analysis GENEVESTIGATOR software was used. Expression data of $O s U S P$ s from Affymetrix rice genome array platform was fetched from GENEVESTIGATOR experiment dataset and analysed with Perturbation tool. Fold change of genes in response to various abiotic stresses was log transformed and expression change was represented in a heat map. Red and green colour represents up-regulation and downregulation of genes respectively.

\section{Results and Discussion}

\section{Identification of $O s U S P s$}

HMMER and BLAST search was employed to identify putative USP genes in rice genome. Together HMMER and BLAST search identified 60 protein models containing USP domain. Based on locus information of the proteins, they were found to be coded by 43 loci. Thus, 43 non redundant OsUSPs were identified in rice genome, named OSUSPs1 to OsUSP43 according to chromosomal location in descending order (Table 1). Apart from USP domain, several OSUSP also contain kinase domain. OsUSP8, OsUSP9, OsUSP10, OsUSP15, OsUSP16, OsUSP21, OsUSP28, OsUSP29, OsUSP32, OsUSP33, OsUSP35, OsUSP36 and OsUSP38 have kinase domain in addition to USP domain, suggesting their putative role in signal transduction and activation of other protein function.

\section{Phylogenetic analysis}

The evolutionary history of both promoter sequence and $O S U S P$ protein sequences were inferred by neighbour joining method (Fig. 1). After eliminating positions with gaps, a total of 650 positions were considered for calculation of evolutionary distance in promoter sequences. In case of protein sequences 105 amino acid positions were considered for distance matrix calculation. Phylogenetic analysis of OsUSP protein and promoter sequences revealed there is no correlation in evolutionary relationship between protein and promoter sequences. Promoter sequences were grouped into six distinct clades, whereas OSUSP protein sequences were grouped into four clades. Chromosomal distribution of OsUSPs revealed their non-uniform presence across the chromosomes (Fig. 2). There are 7 OsUSPs present in chromosome1 and 5; 8 OsUSPs on Chromosome 2; 6 in chromosome $3 ; 1$ on 
chromosome 9 and 11; 5 on chromosome 12. Chromosome 4 and 8 do not have any OsUSP. Tandem duplication of OsUSP genes were found on chromosome 2, chromosome 9 and chromosome 12 .

\section{Digital gene expression}

Expression of OsUSPs in response to various abiotic stresses was analysed using GENEVESTIGATOR software (Fig. 3). We conducted differential expression of $O s U S P$ s in response to anoxia, dehydration high temperature and cold. Expression pattern of $O s U S P$ s differ in various stresses. OsUSP32 (LOC_Os07g47620) was found to be highly upregulated during all stresses examined except cold stress, whereas LOC_Os03g19270 (OsUSP17) was upregulated in dehydration and cold stress. LOC_Os02g47840 (OsUSP12) showed upregulation in response to dehydration stress but it was highly downregulated during anoxia, suggesting its differential mechanism of action in different stresses.

Expression of seven OsUSPs, LOC_Os02g $54590 \quad$ (OsUSP15), LOC_Os01g39970 (OsUSP4), LOC_Os02g12660 (OsUSP9), LOC_Os03g40130 (OsUSP19), LOC_Os12g $07990 \quad$ (OsUSP39), LOC_Os12g08060 (OsUSP40), LOC_Os10g32590 (OsUSP36) was not changed in response to any abiotic stresses, indicating their no role in stress response.OSUSP1 was found to be downregulated in all stresses except high temperature stress, where it is slightly upregulated.

Fig.1 Phylogenetic tree of OsUSP promoter (A) and protein (B) sequences. Neighbour-Joining method was employed for tree reconstruction

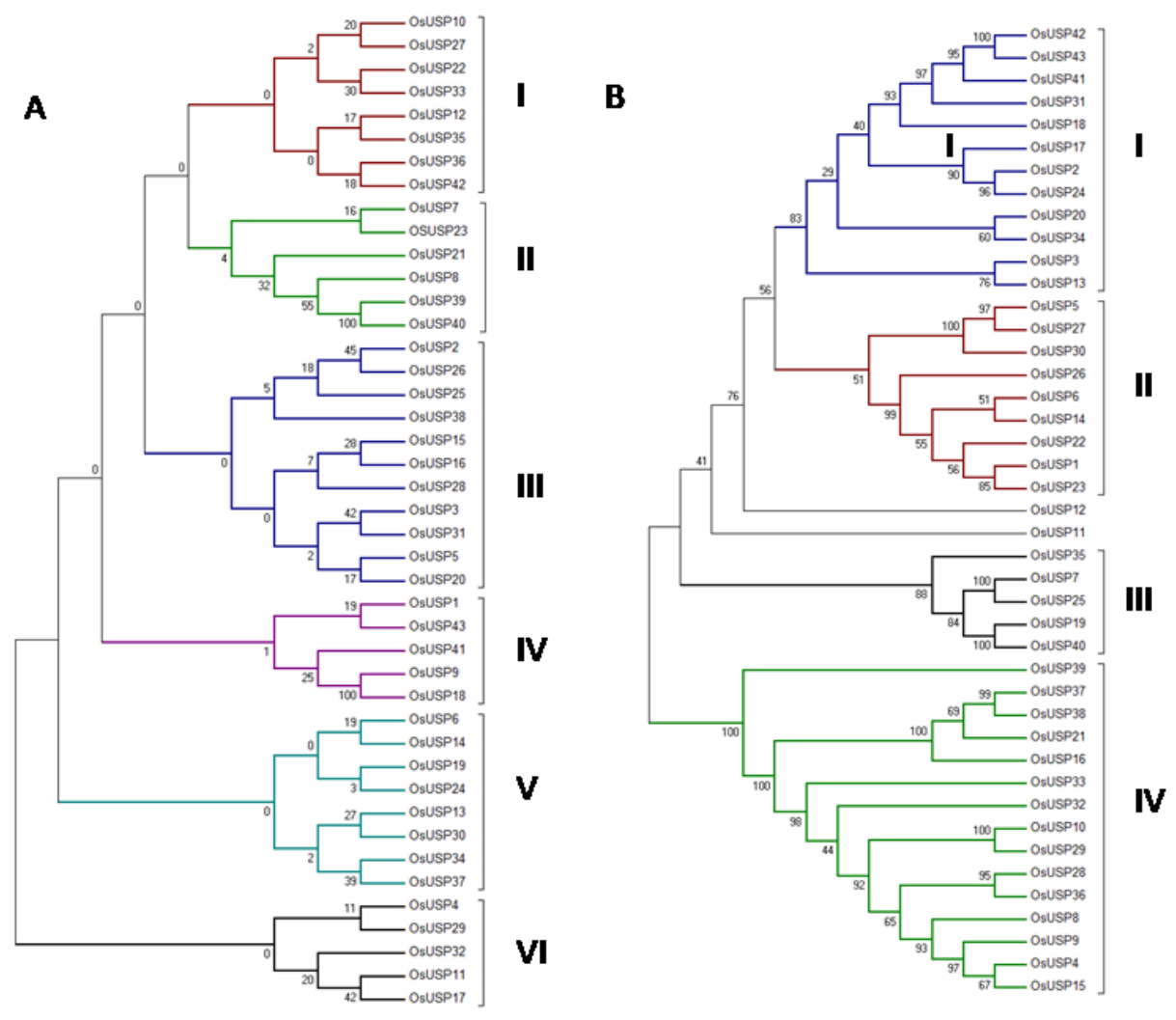


Fig.2 Chromosomal distribution of $O s U S P$ genes. Left bar represent scale in Megabase (Mb)

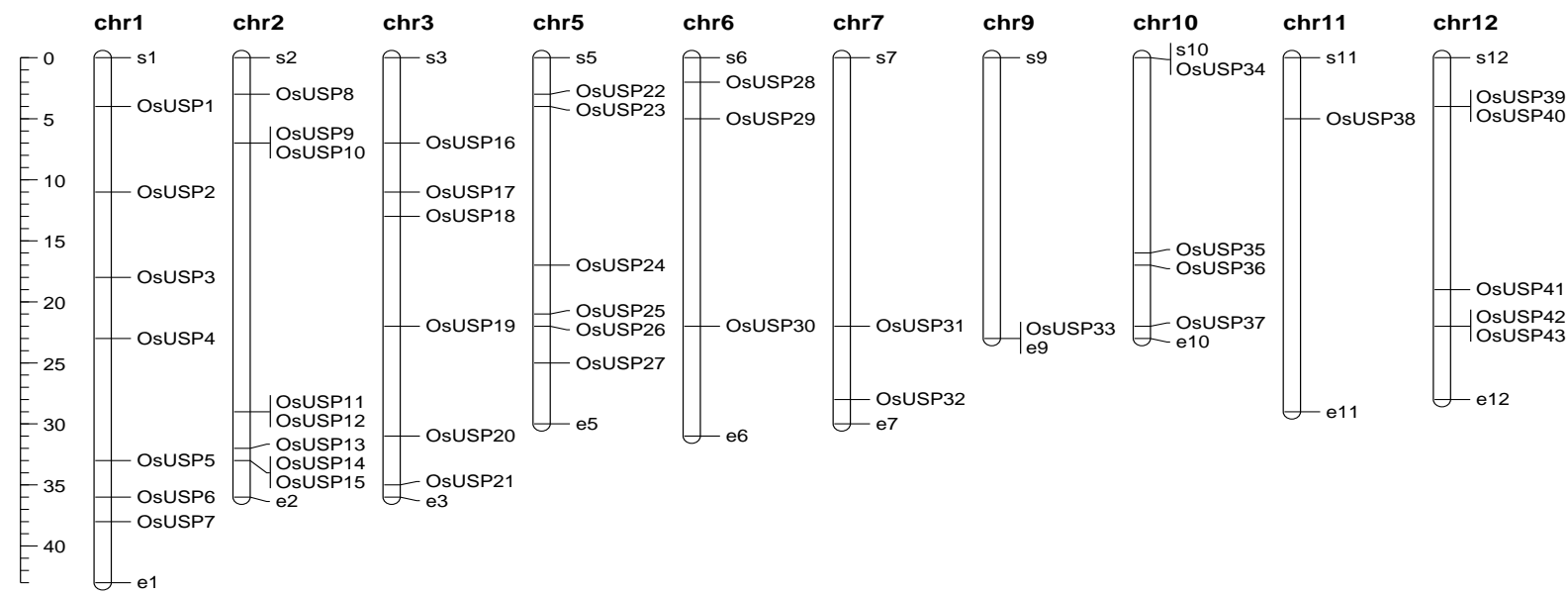

Fig.3 Expression analysis $O s U S P$ genes in response to various abiotic stresses. Heat map of expression fold change was created using GENEVESTIGATOR software

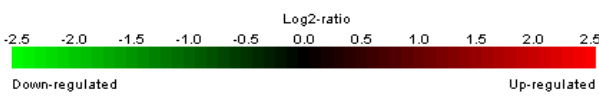

Oryza sativa (10)

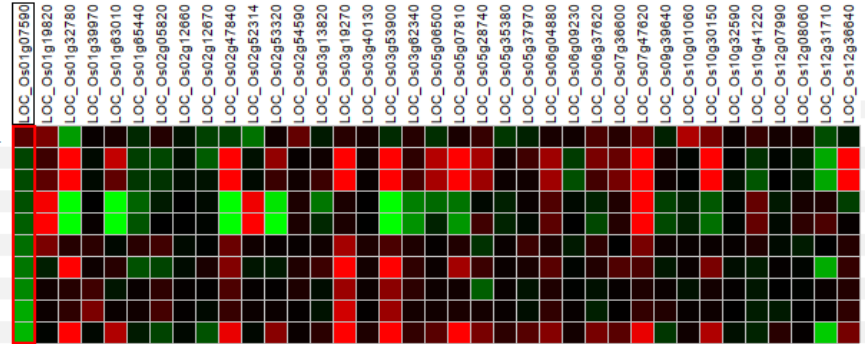

OS-00037 temperature study 1 (Minghui $63 ; 31^{\circ} \mathrm{C}$ ) / untreated calli samples (Minghu ... OS-00225 dehydration study 3 (Dhagaddeshi; $6 \mathrm{~h}$ ) / untreated seedling samples (Dh ... OS-00225 dehydration study 3 (Dhagaddeshi; 3h) / untreated seedling samples (Dh OS-00051 anoxia study 2 (N2 for $12 \mathrm{~h}$ )/ anoxia study 2 (N2 for $1 \mathrm{~h}$ )

OS-00051 anoxia study 2 (N2 for $24 \mathrm{~h}$ )/ anoxia study 2 (N2 for $1 \mathrm{~h}$ )

OS-00102 cold study $4(\mathrm{C} 418 ; 2 \mathrm{~h}) /$ untreated shoot samples (C418)

OS-00225 dehydration study 3 (IR20; 3 h) / untreated seedling samples (IR20)

Os-00102 cold study $4(\mathrm{C} 418,6 \mathrm{~h}) /$ untreated shoot samples (C418)

OS-00102 cold study $4(\mathrm{C} 418 ; 12 \mathrm{~h}) /$ untreated shoot samples (C418)

OS-00225 dehydration study 3 (IR20; 6h) / untreated seedling samples (IR20)

Fig.4 Number of different transcription factor binding site in OsUSP promoters. $\mathrm{X}$ axis represents number of TFBS

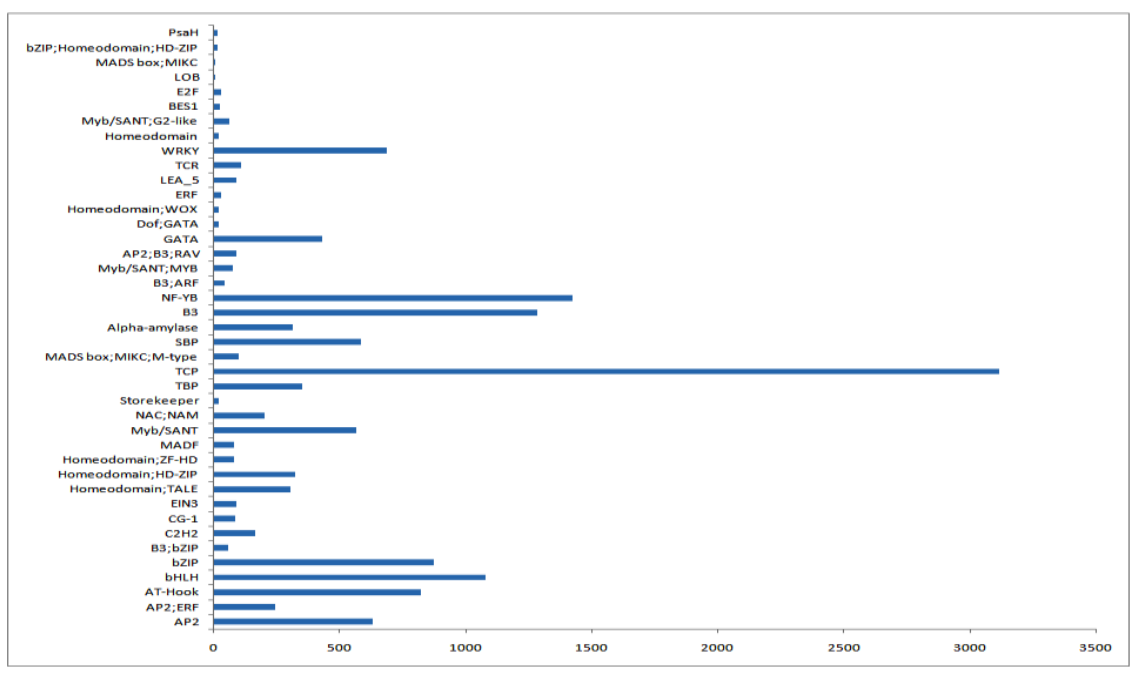


Table.1 Locus id of $O s U S P$ genes

\begin{tabular}{|l|l|l|l|l|l|}
\hline USP gene & Locus id & Chromosome & USP gene & Locus id & Chromosome \\
\hline OsUSP1 & LOC_Os01g07590 & 1 & OsUSP23 & LOC_Os05g07810 & 5 \\
\hline OsUSP2 & LOC_Os01g19820 & 1 & OsUSP24 & LOC_Os05g28740 & 5 \\
\hline OsUSP3 & LOC_Os01g32780 & 1 & OsUSP25 & LOC_Os05g35380 & 5 \\
\hline OsUSP4 & LOC_Os01g39970 & 1 & OsUSP26 & LOC_Os05g37970 & 5 \\
\hline OsUSP5 & LOC_Os01g57450 & 1 & OsUSP27 & LOC_Os05g42230 & 5 \\
\hline OsUSP6 & LOC_Os01g63010 & 1 & OsUSP28 & LOC_Os06g04880 & 6 \\
\hline OsUSP7 & LOC_Os01g65440 & 1 & OsUSP29 & LOC_Os06g09230 & 6 \\
\hline OsUSP8 & LOC_Os02g05820 & 2 & OsUSP30 & LOC_Os06g37620 & 6 \\
\hline OsUSP9 & LOC_Os02g12660 & 2 & OsUSP31 & LOC_Os07g36600 & 7 \\
\hline OsUSP10 & LOC_Os02g12670 & 2 & OsUSP32 & LOC_Os07g47620 & 7 \\
\hline OsUSP11 & LOC_Os02g47650 & 2 & OsUSP33 & LOC_Os09g39640 & 9 \\
\hline OsUSP12 & LOC_Os02g47840 & 2 & OsUSP34 & LOC_Os10g01060 & 10 \\
\hline OsUSP13 & LOC_Os02g52314 & 2 & OsUSP35 & LOC_Os10g30150 & 10 \\
\hline OsUSP14 & LOC_Os02g53320 & 2 & OsUSP36 & LOC_Os10g32590 & 10 \\
\hline OsUSP15 & LOC_Os02g54590 & 2 & OsUSP37 & LOC_Os10g41220 & 10 \\
\hline OsUSP16 & LOC_Os03g13820 & 3 & OsUSP38 & LOC_Os11g08950 & 11 \\
\hline OsUSP17 & LOC_Os03g19270 & 3 & OsUSP39 & LOC_Os12g07990 & 12 \\
\hline OsUSP18 & LOC_Os03g22390 & 3 & OsUSP40 & LOC_Os12g08060 & 12 \\
\hline OsUSP19 & LOC_Os03g40130 & 3 & OsUSP41 & LOC_Os12g31710 & 12 \\
\hline OsUSP20 & LOC_Os03g53900 & 3 & OsUSP42 & LOC_Os12g36630 & 12 \\
\hline OsUSP21 & LOC_Os03g62340 & 3 & OsUSP43 & LOC_Os12g36640 & 12 \\
\hline OsUSP22 & LOC_Os05g06500 & 5 & & & \\
\hline & & & & & \\
\hline
\end{tabular}

\section{Promoter analysis}

Promoter region of gene is essential to drive expression of the genes. We found there is significant differential expression of OsUSP genes in response to various abiotic stresses as described above. In order to analyse promoter region of OsUSPs, we have retrieved $1 \mathrm{~Kb}$ upstream sequence of respective OSUSP transcripts and this $1 \mathrm{~Kb}$ region was considered as putative promoters of the genes. After scanning of promoter regions in PlantPAN database, we found 14533 transcription factor binding site across all the OsUSP promoter (Fig. 4). Forty one different transcription factors were found to bind with this TFBS. In our study we found highest number of TFBS for TCP (3118) transcription factor, whereas lowest number of TFBS was found for LOB and MIKC transcription factor. Both of these TFs have only six TFBS each in 43 OsUSP promoters. NF-YB, B-ZIP, B3, MYB, NAC, bHLH, AP2, WRKY and AT-Hook transcription factor binding sites were overrepresented in the OsUSP promoters. TCP, NF-YB and B3 TFBS were found to be present in all the OsUSPs.

In this present study 43 OsUSP genes were identified in rice genome and their promoter region was analysed to see which TFs bind with the promoters. Phylogenetic analysis of USP protein and promoter was done 
separately and no correlation was found between NJ tree of OsUSP proteins and promoters. We found 14533 different transcription factors binding site in 43 OsUSPs, which belong to $41 \mathrm{TF}$ family. Among these TFs, TCP, B-ZIP, WRKY, bHLH, GATA is already reported to be involved in various abiotic stress responses.

\section{References}

Bhuria, M., Goel, P., Kumar, S. and Singh, A.K., 2016. The promoter of AtUSP is co-regulated by phytohormones and abiotic stresses in Arabidopsis thaliana. Frontiers in plant science, 7, p.1957.

Chou, M.X., Wei, X.Y., Chen, D.S. and Zhou, J.C., 2007. A novel nodule-enhanced gene encoding a putative universal stress protein from Astragalussinicus. Journal of plant physiology, 164(6), pp.764-772.

Isokpehi, R.D., Simmons, S.S., Cohly, H.H., Ekunwe, S.I., Begonia, G.B. and Ayensu, W.K., 2011. Identification of drought-responsive universal stress proteins in viridiplantae. Bioinformatics and biology insights, 5, pp.BBI-S6061.

Jung, Y.J., Melencion, S.M.B., Lee, E.S., Park, J.H., Alinapon, C.V., Oh, H.T., Yun, D.J., Chi, Y.H. and Lee, S.Y., 2015. Universal stress protein exhibits a redox-dependent chaperone function in Arabidopsis and enhances plant tolerance to heat shock and oxidative stress. Frontiers in plant science, 6, p.1141.

Kerk, D., Bulgrien, J., Smith, D.W. and Gribskov, M., 2003. Arabidopsis proteins containing similarity to the universal stress protein domain of bacteria. Plant Physiology, 131(3), pp. 1209-1219.

Li, W.T., Wei, Y.M., Wang, J.R., Liu, C.J., Lan, X.J., Jiang, Q.T., Pu, Z.E. and Zheng, Y.L., 2010. Identification, localization, and characterization of putative USP genes in barley. Theoretical and Applied Genetics, 121(5), pp.907-917.

Loukehaich, R., Wang, T., Ouyang, B., Ziaf, K., Li, H., Zhang, J., Lu, Y. and Ye, Z., 2012. SpUSP, an annexin-interacting universal stress protein, enhances drought tolerance in tomato. Journal of experimental botany, 63(15), pp.55935606.

Loukehaich, R., Wang, T., Ouyang, B., Ziaf, K., Li, H., Zhang, J., Lu, Y. and Ye, Z., 2012. SpUSP, an annexin-interacting universal stress protein, enhances drought tolerance in tomato. Journal of experimental botany, 63(15), pp.55935606.

Maqbool, A., Zahur, M., Husnain, T. and Riazuddin, S., 2009. GUSP1 and GUSP2, two drought-responsive genes in Gossypiumarboreum have homology to universal stress proteins. Plant molecular biology reporter, 27(1), pp.109-114

Nachin, L., Nannmark, U. and Nyström, T., 2005. Differential roles of the universal stress proteins of Escherichia coli in oxidative stress resistance, adhesion, and motility. Journal of bacteriology, 187(18), pp.6265-6272.

Ndimba, B.K., Chivasa, S., Simon, W.J. and Slabas, A.R., 2005. Identification of Arabidopsis salt and osmotic stress responsive proteins using two- dimensional difference gel electrophoresis and mass spectrometry. Proteomics, 5(16), pp. 4185-4196.

Persson, Ö., Valadi, A., Nyström, T. and Farewell, A., 2007. Metabolic control of the Escherichia coli universal stress protein response through fructose- 6- phosphate. Molecular microbiology, 65(4), pp.968-978.

Sauter, M., Rzewuski, G., Marwedel, T. and Lorbiecke, R., 2002. The novel 
ethylene- regulated gene OsUsp1 from rice encodes a member of a plant protein family related to prokaryotic universal stress proteins. Journal of Experimental Botany, 53(379), pp.23252331.

Shinozaki, K. and Yamaguchi-Shinozaki, K., 2007. Gene networks involved in drought stress response and tolerance. Journal of experimental botany, 58(2), pp.221-227.

Sousa, M.C. and McKay, D.B., 2001. Structure of the universal stress protein of Haemophilus influenzae. Structure, 9(12), pp.1135-1141.

Tkaczuk, K.L., A. Shumilin, I., Chruszcz, M., Evdokimova, E., Savchenko, A. and Minor, W., 2013. Structural and functional insight into the universal stress protein family. Evolutionary applications, 6(3), pp.434-449.

Udawat, P., Mishra, A. and Jha, B., 2014. Heterologous expression of an uncharacterized universal stress protein gene (SbUSP) from the extreme halophyte, Salicornia brachiata, which confers salt and osmotic tolerance to E. coli. Gene, 536(1), pp.163-170.

Zarembinski, T.I., Hung, L.W., MuellerDieckmann, H.J., Kim, K.K., Yokota, H., Kim, R. and Kim, S.H., 1998. Structure-based assignment of the biochemical function of a hypothetical protein: a test case of structural genomics. Proceedings of the National Academy of Sciences, 95(26), pp.1518915193.

\section{How to cite this article:}

Rakesh Bhowmick. 2019. Comprehensive Analysis of Universal Stress Proteins and Their Promoter Sequences in Rice. Int.J.Curr.Microbiol.App.Sci. 8(07): 1279-1286. doi: https://doi.org/10.20546/ijcmas.2019.807.152 